\title{
Motivation in question
}

'There are signs that today's young adults may be the first generation in modern history to be less healthy than their parents. Respiratory diseases and cancers, diabetes and obesity, heart and liver disease, and some psychological problems, such as depression, are all strongly linked to health behaviours and lifestyle. A majority of the maladies that now cause people to consult health care professionals ... are largely preventable or remediable through health behaviour change."

In that spirit of commitment to continuing professional development which is expected of today's GP, I recently attended a 2-day course in Motivational Interviewing (MI) to empower me to implement the new 'NHS Health Check' currently being 'rolled out' around the country. This stimulating experience led me to look up the work of the pioneers of $\mathrm{MI}$ in the UK. The opening sentences of their introductory handbook for healthcare practitioners are quoted above. While the course provided helpful tips to improve consultation skills (notably in favouring an 'asking, listening, informing' approach rather than a 'lecturing, arguing, warning' style), these three sentences reinforced my determination to resist any inclination to attempt to change my patients' behaviour.

The first sentence sounds a familiar note of apocalyptic gloom. In the prevailing culture of pessimism and despair, this preposterous foundational assumption of the campaign to extend $\mathrm{Ml}$ in primary health care is so widely accepted that it requires no elaboration or supporting evidence. It is evidently contradicted by that other great contemporary panic about the burden on society that is anticipated as more and more members of this generation become centenarians.

The second sentence affirms another familiar prejudice: if you get ill, it's your own fault. But it is simply not true that even most of the diseases mentioned are the result of individual behaviour. Apart from the link between smoking and lung cancer (the incidence of which has been declining for decades), lifestyle factors are of negligible significance in most common cancers and this is also true of most cases of respiratory, heart, and liver disease. What about neurological and rheumatological problems, such as Parkinson's disease, Alzheimer's, multiple sclerosis, rheumatoid arthritis, connective tissue disorders? Why not focus on chronic mental illnesses, such as schizophrenia and bipolar affective disorder? It seems that promoters of $\mathrm{MI}$, like politicians and public health authorities, are only interested in conditions that offer scope for victimblaming and moralising.

The third sentence asserts the dubious proposition that virtuous living can prevent or cure most illness. Apart from the well substantiated benefits of stopping smoking, the evidence that other lifestyle changes - notably in relation to diet and exercise - make a significant difference to health is poor. The evidence is even weaker that any of the methods used to promote such changes in lifestyle consistently achieves the desired outcomes.

The Ml handbook and the same authors' recent promotional feature in the BMJ claim that $\mathrm{MI}$ 'outperformed traditional advice giving in $80 \%$ of studies'. ${ }^{2}$ Given that 'traditional advice giving' is well known to be useless if not counterproductive, this is not as impressive a claim as it appears on first glance.

The fact that $\mathrm{Ml}$ originated in the development of counselling techniques for dealing with problems of addiction to alcohol and drugs in the 1980s gives little grounds for optimism for its expansion into the world of primary care and chronic disease management. My experience of attempting to treat addiction in general practice led me to the conclusion that the way forward lay through doctors concentrating on the diagnosis and treatment of disease and leaving questions of lifestyle and behaviour to the only people who can resolve them - our patients.

\section{REFERENCES}

1. Rollnick S, Miller WR, Butler CC. Motivational Interviewing in health care: helping patients change behaviour. New York: The Guilford Press, 2008.

2. Rollnick S, Butler CC, Paul Kinnersley P, et al. Motivational interviewing. BMJ 2010 Apr 27; 340 c1900. doi: 10.1136/bmj.c1900.

DOI: 10.3399/bjgp10X514963 